\title{
Subacute Combined Degeneration (SACD), Is it a cause of diffusion restriction of the Spinal cord? A Case Report with literature review
}

\author{
Abeer Safan ${ }^{1}$, Mayada Ahmed ${ }^{1}$, Ali AlSalahat ${ }^{1}$, Ahmed ElSetouhy ${ }^{1}$, and Ahmed own ${ }^{1}$ \\ ${ }^{1}$ Hamad Medical Corporation
}

February 11, 2022

\begin{abstract}
Subacute combined degeneration (SACD) of the spinal cord is a common neurological manifestation of vitamin B12 deficiency. We report a rare finding of hyperintense T2 signal in the dorsal and lateral columns with restricted diffusion in MR study of the thoracic spine in 47 years old diagnosed with SACD.
\end{abstract}

Subacute Combined Degeneration (SACD), Is it a cause of diffusion restriction of the Spinal cord? A Case Report with literature review

Authors:

Abeer Sabry Safan ${ }^{1}$, Ali Al-Salahat ${ }^{1}$, Mayada Ahmed, ${ }^{2}$ Ahmed El Sotouhy ${ }^{2}$, Ahmed Own ${ }^{2}$

${ }^{1}$ Department of Neurology, Neurosciences Institute, Hamad Medical Corporation, Doha, Qatar.

${ }^{2}$ Department of Neuroradiology, Neurosciences Institute, Hamad Medical Corporation, Doha, Qatar.

${ }^{3}$ Department of Clinical Radiology, WCM-Q.

Correspondence:

Dr. Ahmed Hassan Aly El Sotouhy

Hamad Medical Corporation Doha, Qatar

Email: aelsetouhy@hamad.qa

\section{ABSTRACT:}

Subacute combined degeneration (SACD) of the spinal cord is a common neurological manifestation of vitamin B12 deficiency. We report a rare finding of hyperintense T2 signal in the dorsal and lateral columns with restricted diffusion in MR study of the thoracic spine in 47 years old diagnosed with SACD.

Keywords: diffusion-weighted imaging (DWI), B12 deficiency, subacute combined degeneration of the spinal cord (SACD).

\section{Key Clinical Message:}

Diffusion restriction (DWI) is not well studied in nutritional deficiencies with spinal cord involvement, such as vitamin B12 deficiency. We advocate that spinal cord DWI may be a beneficial magnetic resonance sequence for the diagnosis of SACD. Conversely, more studies are required to correlate and establish its relevance.

\section{Introduction:}


Subacute combined cord degeneration of the spinal cord is a type of myelopathy that most commonly manifests due to vitamin B12 deficiency. The classical clinical presentation consists triad of symmetrical diminished proprioception, sensory ataxia with pyramidal signs. 5

The commonly reported neuroimaging finding is symmetrical high T2 signal on spinal cord MRI. 1 The presence of High signal DWI with diffusion restriction is reported in one previous case report.2

This paper aims to describe the radiological features on spinal cord DWI in 47 years-old patient presenting with subacute ataxic symptoms diagnosed to have SACD, which can have overlapping features with other myelopathies spinal cord ischemic diseases.

\section{Case presentation:}

Here we present a 47 years old, right-handed male, who is a strict vegetarian, without known past medical or surgical history, who presented to the emergency department of our hospital with sudden onset three days history of unsteady gait with imbalance associated with bilateral lower limb numbness, from the soles of the feet to his knees. He denied any back or neck pain, with no urinary symptoms [No urinary or fecal incontinence or retention], no numbness in the saddle area. He has had no similar episodes in the past, with no Fever or constitutional symptoms. There was no history of preceding trauma. The patient denied taking any supplements or exposure to heavy metals.

Upon initial assessment, his vitals were RR 20, BP 14578, HR 84, 98\% on RA, Temp 36.9 degrees. Complete neurological exam yielded higher mental function, intact, alert, conscious, and oriented to time, place, and person. Cranial nerve examination, including a fundus exam, was normal with no nystagmus. Motor exam of upper/lower limb normal 5/5 as per MRC grade, with deep tendon reflexes (DTR) +2 in the upper limb, and +1 in the ankle, with flexor plantar. The sensory exam showed an impaired sense of proprioception and vibration to the knee, intact in the upper limb, and the rest of the sensory modality intact with no sensory level. Cerebellar examination showed cautious gait with significantly impaired lower limb coordination, evident by dysmetria and impaired heel to chin. There were no detectable cerebellar signs in the upper limbs. Special tests revealed positive Romberg's sign, with negative Spurling's and straight leg raise or lasègue sign. The findings above were more suggestive of sensory ataxia.

Initial Laboratory assessment showed normal hemoglobin of 13.6, with hyperchromic, macrocytosis of red blood cells on peripheral smear, mean corpuscular volume, mean corpuscular hemoglobin, $105.7 \mathrm{fl}$, and 36.8 pg, 34.8 respectively. A plain non-enhanced computed tomography (CT) head was unremarkable. The patient was given an emergency Intra-muscular B12 $1000 \mathrm{mcg} / \mathrm{ml}$ injection before further blood samples were taken for B12 levels. Hence, B12 level was falsely elevated of a value $1476 \mathrm{pg} / \mathrm{ml}(187-1,058 \mathrm{pg} / \mathrm{ml})$. However, the rest of the work showed a normal folate level of 76 and an elevated homocysteine level of 78.0, correlating with Vitamin B12 deficiency. Pernicious anemia screening was unrevealing, and it was attributed to his vegan diet.

Magnetic resonance imaging (MRI) of the head and whole spine was performed. It showed lower thoracic spinal cord posterior para-median relatively symmetrical intramedullary areas of signal abnormality extending opposed D11 and D12 vertebral levels high T2 signal intensity associated with restricted diffusion pattern in the DWI that raised the possibility of subacute cord ischemia vs. subacute combined degeneration secondary to vitamin B12 deficiency. Noted also Multilevel cervical posterior disc bulges with partial neural compromise are more significant at the C5-6 level. However, this does not correlate with his current presentation.

Diagnosis of subacute combined degeneration of the spinal cord was made, and he was placed on intramuscular methylcobalamin (1000 $\mu \mathrm{g}$ daily) for five days. During his admission, he showed improvement and ambulating with minimal assistance. The patient was discharged home on oral 2000mg Q-weekly B12 supplements and regular follow-up, which the patient has numbness has resolved and was able to walk without any assistance.

\section{Discussion:}

Subacute combined degeneration (SACD) of the spinal cord is the most frequent central neurological man- 
ifestation of vitamin B12 deficiency, disrupting the myelination process causing degeneration of the spinal cord's dorsal and lateral white matter. [3] This usually contributes to a slow progressive paraparesis, symmetric dysesthesia, disruption of proprioception that can lead to sensory ataxia. 3-4 In more advanced cases, corticospinal tract involvement can cause abnormal deep tendon reflex, ultimately, spastic paraparesis and tetra-paresis in some cases, with autonomic dysfunction manifesting as urinary incontinence and sexual dysfunction. 4

Neurological manifestation in B12 deficiency in the absence of anemia is not uncommon, as our patient's blood count showed macrocytosis with normal hemoglobin indices.

SACD is usually a gradually progressive process; however, sudden onset presentation warrants further workup to rule out mimics, such as spinal cord ischemia with posterior spinal artery involvement, which is uncommon due to the dual nature of these arteries and pial collateral network. 8

Since the early 1990s, MRI has been crucial in diagnosing vitamin B12-deficiency related central nervous system (CNS) - related complications and most conspicuously to rule out mimics, such as infectious and none infectious myelitis,

acute/subacute ischemic, or even demyelinating disease like multiple sclerosis. [4] SACD has typical radiological MRI findings, with a symmetrical high T2 signal intensity, confined mainly in the dorsal/posterior and lateral columns of cervical and thoracic spins, with an inverted V sign due to symmetrical involvement the structures mentioned above. [5]

Polat et al. conducted a retrospective study on 37 patients with biochemical evidence of B12 deficiency, showed significantly increased apparent diffusion coefficient (ACD) values in multiple brain areas such as the amygdala, hypothalamus, and striate cortex, but did not further look at radiological features in SACD. 1

Han Gao et al., in 2021, conducted a retrospective study on reactional Nitrous oxide (NO) related SACD compared to other SACD, showed MRI manifestation was more common among SACD-related NO with T2WI- high signal intensity in cervical and thoracic segments of the spinal cord, bearing in mind that Nitric oxide users had lower B12 levels. 7

Our patient had a sudden onset presentation that worsened over three days, of symmetrical dysesthesia and impaired sense of proprioception and vibration, found to have macrocytosis on peripheral smear with raised homocysteine level correlating with B12 deficiency without anemia.

His MRI showed an inverted V sign and high signal in thoracic spine segments on DWI sequence; that his symptoms resolved with adequate B12 replacement.

\section{Conclusion:}

The presence of Diffusion restriction and the typical high T2 signal intensity of the spinal cord on MR Imaging in Patients with Vitamin B12 deficiency has an additional value in confirming the diagnosis of Subacute Combined Degeneration (SACD). It needs to be involved in the routine MR imaging protocol of these patients.

\section{Acknowledgements}

None.

\section{Consent}

Written consent

\section{Conflicts of Interest}

The authors have no conflict of interest to disclose.

\section{Funding}


Qatar National Library funded the open access publication fees of this case.

\section{Author contributions}

Writing the initial draft of the manuscript: AS, AA

Conceptualization and supervision: $\mathrm{MA}, \mathrm{AE}$

Medical management of the case: AA

Revising the manuscript critically and literature review: AS, MA, AE

\section{Appendix 1}

\begin{tabular}{lll}
\hline Name & Location & Contribution \\
\hline Abeer Sabry Safan & Neurosciences Institute, Hamad Medical Corporation, Doha, Qatar & Writing the init \\
Ali Al-Salahat & Neurosciences Institute, Hamad Medical Corporation, Doha, Qatar & Medical manag \\
Mayada Ahmed & Neuroradiology, Neurosciences Institute, Hamad Medical Corporation, Doha, Qatar & Obtaining the r \\
Ahmed El Sotouhy & Neuroradiology, Neurosciences Institute, Hamad Medical Corporation, Doha, Qatar & Conceptualizati \\
Ahmed Own & Neuroradiology, Neurosciences Institute, Hamad Medical Corporation, Doha, Qatar & Conceptualizati \\
\hline
\end{tabular}

\section{References}

1. Polat HB, Kanat A, Celiker FB, Tufekci A, Beyazal M, Ardic G, et al. Rationalization of using the MR diffusion imag:

2. Tian C. Hyperintense signal on spinal cord diffusion-weighted imaging in a patient with subacute combined degeneratic

3. Saji AM, De Jesus O. Spinal cord subacute combined degeneration. In: StatPearls. Treasure Island (FL): StatPearls P

4. Ralapanawa DMPUK, Jayawickreme KP, Ekanayake EMM, Jayalath WATA. B12 deficiency with neurological manifest

5. Briani C, Dalla Torre C, Citton V, Manara R, Pompanin S, Binotto G, et al. Cobalamin deficiency: clinical picture an

6. Gaillard F. Subacute combined degeneration of the cord [Internet]. Radiopaedia.org. [cited 2021 Apr 28]. Available fro

7. Gao H, Li W, Ren J, Dong X, Ma Y, Zheng D. Clinical and MRI differences between patients with subacute combined 8. Vuillier F, Tatu L, Camara A, Muzard E, Moulin T. Unusual sensory disturbances revealing posterior spinal artery infe

\section{Hosted file}

B12images.docx available at https://authorea.com/users/383880/articles/556122-subacutecombined-degeneration-sacd-is-it-a-cause-of-diffusion-restriction-of-the-spinal-cord-acase-report-with-literature-review 